\title{
The Dynamic Characteristic of a Faulted Rotor System with Multi-Objective Optimization Designed SFD
}

\author{
Liang Ma ${ }^{1}$, Jun Wang ${ }^{2,3, *}$ and Guichang Zhang ${ }^{1}$ \\ 1 College of Aeronautical Engineering, Civil Aviation University of China, Tianjin 300300, China \\ 2 State Key Laboratory of Engines, Tianjin University, Tianjin 300072, China \\ 3 Renai College, Tianjin University, Tianjin 301636, China \\ * Correspondence: wjun@tju.edu.cn; Tel.: +86-022-6857-9990
}

Received: 31 July 2019; Accepted: 19 August 2019; Published: 3 September 2019

\begin{abstract}
As an important part of the turbomachinery, the rotor-bearing system has been upgraded to provide a high rotating speed in order to meet the demand of high power production. With increasing demand for stability, the squeeze film damper (SFD) has been widely used in industrial machinery because it can reduce the vibration amplitude and suppress the external force. Usually, it shows inadaptability under the different working conditions where the SFD parameters didn't change appropriately. Therefore, the reasonable choice of operational parameters of SFD is the key solution that can provide viscous damping effectively and restrain the nonlinear vibration generated by faults. In this paper, the mathematical model of a rotor-ball bearing-SFD system considering the misalignment fault and misalignment-rubbing coupling fault is built first. Then the dynamic characteristics under typical working conditions $(\omega=1000 \mathrm{rad} / \mathrm{s})$ of the faulted rotor are discussed. The vibration attenuation effects of the SFD parameters selected by using the multi-objective optimization method on the dynamic responses are analyzed. The results show that when the rotor system operates under different states, the value and the sensitivity of optimization parameters are altered. With no fault, the amplitude of fundamental frequency decrease $23 \%$. With the misalignment fault, the amplitude of the fundamental frequency decreases by $43.4 \%$, the amplitude of $2 \times$ fundamental frequency decreases by $27.5 \%$, and the amplitude of $3 \times$ fundamental frequency decreases by $66.7 \%$. With the misalignment-rubbing coupling fault, the amplitude of fundamental frequency reduces by $7.4 \%$, the amplitude of $2 \times$ fundamental frequency drops by $51.5 \%$, and the amplitude of $3 \times$ fundamental frequency drops by $16.8 \%$. Overall, the feasibility of the optimization method of the variable-structured SFD operational parameters for the faulted rotor system is verified. These parametric analyses are very helpful in the development of a high-speed rotor system and provide a theoretical reference for the vibration control and optimal design of rotating machinery.
\end{abstract}

Keywords: rotor; SFD; misalignment rubbing coupling faults; multi-objective optimization

\section{Introduction}

The rotor bearing system of the turbomachinery, such as turbines, pumps and compressors, might undergo nonlinear supported forces and fault excitations. With increasing demand for vibration and stability, the squeeze film damper has been widely used in industrial machinery [1].

In 1977, based on Reynolds equation of short bearing assumption, Humes [2] studied the motion law of rigid rotor system with SFD, and the theoretical model of the hydrodynamic oilfilm force was proposed in 1978. Meanwhile, the obtained results were in agreement with the experimental results; when Coorper [3] presented the experimental study on SFD, and applied it to the Conway engine successfully, the vibration amplitude of the system was reduced by $60 \%$. Considering the effect of the cavitation, Ruggiero et al. [4] found the "oil whirl" produced by the non-linear fluid forces. 
Wang [5] studied a controllable SFD for a rotor system and revealed its effect mechanism. Chang [6-8] analyzed the dynamic behavior of a hybrid SFD-mounted gear-bearing system based on the typical unbalance approximation and discussed the rotating speed and damping effects. Inayat-Hussain $[9,10]$ presented the dynamic analysis of a flexible rotor mounted in SFD with and without retaining springs independently, and the results showed that the onset of bifurcation increased with the stiffness of the retainer spring, which provided insights into the effect of the design parameters of SFD on the rotor response. Qin [11] derived the dynamic equations of the single-disk rotor -SFD system under the elastic support and studied the nonlinear characteristics of the vibration responses. Consequently, there is no doubt that the application of SFD in rotor system can enhance the operational stability to a large extent. However, the improvement not only depends on the advanced structure design but also relates to the correlation of SFD parameters and the real condition of the rotor system. In other words, it is important to find out a suitable parameter set of SFD to prevent system instability due to fault occurrence as well as to facilitate product design and vibration control.

In this paper, a dynamic system model of the fault rotor system with SFD is established first. Then the dynamic characteristics under typical working conditions of the faulted rotor are discussed. And the vibration attenuation effects of the oil film gap $\left(C_{S}\right)$, elastic support stiffness $\left(K_{\mathrm{a}}\right)$ and the lubricating oil viscosity $\left(\mu_{\mathrm{s}}\right)$ of SFD selected by using the multi-objective optimization method on the dynamic responses are analyzed.

\section{The Basic Theory}

\subsection{Rotor System}

The simplified model of a SFD-rotor-bearing system supported by deep groove ball bearing is shown in Figure 1. Rotor equivalent lumped mass points are $M_{\mathrm{s} 1}, M_{\mathrm{d} 1}, M_{\mathrm{s} 2}, M_{\mathrm{s} 3}, M_{\mathrm{s} 4}, M_{\mathrm{s} 5}, M_{\mathrm{d} 2}, M_{\mathrm{s} 6}$ respectively, $M_{\mathrm{o} 1}$ and $M_{\mathrm{o} 2}$ are lumped mass points of rolling bearing outer ring. $K$ is stiffness of the flexible shaft; $K_{\mathrm{a}}$ is the supporting stiffness of squirrel cage, $C_{\mathrm{z}}$ and $C_{\mathrm{p}}$ are damping coefficients of rotor in bearing and disk. $\delta$ is the gap between rotor and stator; $e_{1}$ and $e_{2}$ are the eccentricities of the disk; $\alpha_{1}$ and $\alpha_{2}$ are the eccentric angles of the disk; $k_{\mathrm{s}}$ is the stiffness coefficient.

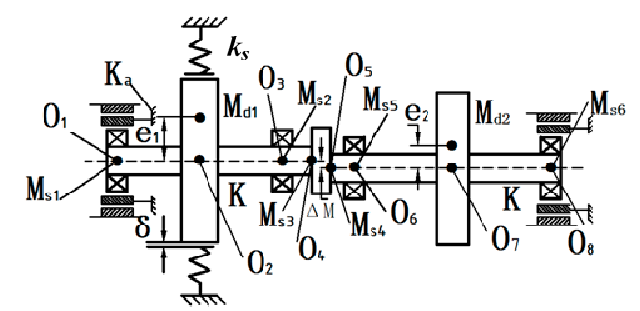

Figure 1. The simplified model of SFD-rotor-bearing system.

According to the Lagrange equation, the differential equation of motion of rotor system can be obtained as follow:

$$
[M]\{\ddot{q}\}+[C]\{\dot{q}\}+[K]\{q\}=\{Q\},
$$

In the formula, $[M],[C],[K]$ are the mass matrix, damping matrix and stiffness matrix, respectively. Their details have been further described in Appendix A.

The system non-dimensional transformation is introduced: $\tau=\omega t,\{\bar{q}\}=\frac{\{q\}}{\mu},\{\dot{\bar{q}}\}=\frac{\{\dot{q}\}}{\mu \omega},\{\ddot{\bar{q}}\}=\frac{\{\ddot{q}\}}{\mu \omega^{2}}$, where $\omega$ is rotational angular velocity of the shaft; $\mu$ is the clearance of rolling bearing. Formula (1) transforms into non-dimensional equation:

$$
\{\ddot{\bar{q}}\}=-\frac{[M]^{-1}[C]}{\omega}\{\dot{\bar{q}}\}-\frac{[M]^{-1}[K]}{\omega^{2}}\{\bar{q}\}+\frac{[M]^{-1}\{\bar{Q}\}}{\mu \omega^{2}} .
$$




\subsection{Rub-Impact Model}

Assuming that the rotor is in a state of rubbing, the friction between the rotor and the stator is in accordance with the Coulomb's law [12].

Set $e=\sqrt{x_{p}{ }^{2}+y_{p}^{2}}$ as radial relative displacement between the rotor disk and the casing, $\delta$ is the gap between the rotor and stator at rest. When $e<\delta$, there is no rub-impact phenomenon. When $e \geq \delta$, rub-impact occurs, normal force $F_{N}$ and Tangential friction force $F_{T}$ can be expressed as:

$$
\left\{\begin{array}{l}
F_{T}=f F_{N} \\
F_{N}=k_{s}(e-\delta) \quad(e \geq \delta)
\end{array} .\right.
$$

In the formula: $k_{s}$ is impact stiffness coefficient, $f$ is friction coefficient.

The rub-impact forces are decomposed in the $x-y$ coordinate system:

$$
\left\{\begin{array}{c}
F_{p x} \\
F_{p y}
\end{array}\right\}=-\frac{F_{N}}{e}\left[\begin{array}{cc}
1 & -f \\
f & 1
\end{array}\right]\left\{\begin{array}{c}
X_{p} \\
Y_{p}
\end{array}\right\}
$$

\subsection{Gear Coupling Model}

Figure 2 shows a schematic diagram of the gear coupling. When the gear teeth are meshing tightly to transmit a large torque, deformation of gear teeth is inevitable and the following meshing force is related to the deformation amount, meshing distance and meshing rigidity [13]. The torque-induced meshing force is given by

$$
\begin{gathered}
F_{T i}=\phi L_{i} K_{L i}, \\
\phi=T / \sum_{i=1}^{z}\left[L_{i} K_{L i}\left(R+L_{i}\right)\right],
\end{gathered}
$$

where $\phi$ is the deformation angle of the gear tooth, $T$ is torque, $L_{i}$ and $K_{L i}$ are the equivalent meshing distance and stiffness of each gear tooth respectively, $R$ is the radius of the root circle.

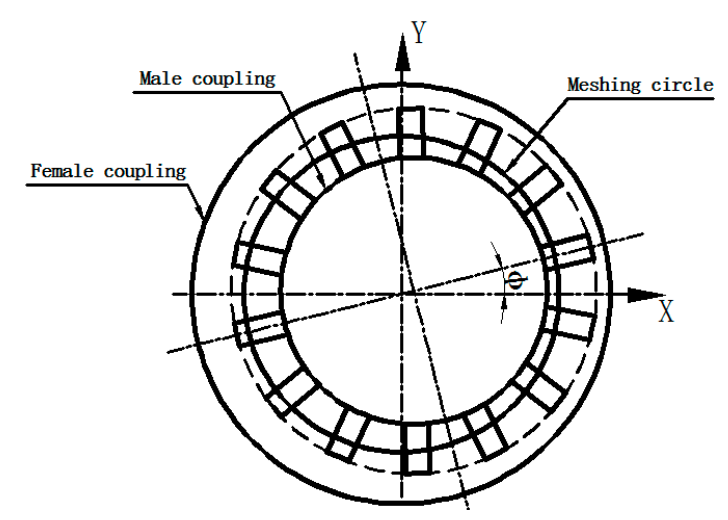

Figure 2. Schematic diagram of the gear coupling.

When the torque is getting transmitted by gear teeth, a relative movement between the two meshing teeth takes place, which is induced by the system vibration at the same time. As a result, the displacement of meshing nodes causes a deformation of each tooth so that another meshing force is produced, which is expressed as:

$$
\begin{aligned}
F_{D i} & =\left(e^{\prime} \sin \varphi_{i}\right) K_{L i}, \\
\varphi_{i} & =2 \pi(i-1) / z \\
e^{\prime} & =\sqrt{x^{2}+y^{2}} .
\end{aligned}
$$


In the formula, $\varphi_{i}$ is the position angle of the gear tooth, and $e^{\prime}$ is the misalignment of gear coupling.

From the above analysis, it can be concluded that the meshing force of coupling misalignment can be obtained as:

$$
F_{L i}=\left(\phi L_{i}+e^{\prime} \sin \varphi_{i}\right) K_{L i}
$$

Then, the reactions of meshing force in the $\mathrm{X}$ and $\mathrm{Y}$ directions of the rotor system are as follows:

$$
\begin{aligned}
& F_{L X}=\sum_{i=1}^{z}\left(\phi L_{i}+e^{\prime} \sin \varphi_{i}\right) K_{L i} \cos \theta_{i}, \\
& F_{L Y}=\sum_{i=1}^{z}\left(\phi L_{i}+e^{\prime} \sin \varphi_{i}\right) K_{L i} \sin \theta_{i} .
\end{aligned}
$$

\subsection{Squeeze Film Damper}

The oil film force of SFD can be calculated according to the theory of hydrodynamic lubrication. The instantaneous pressure distribution can be computed from the incompressible Reynolds equation [14]:

$$
\frac{1}{R_{\mathrm{s}}^{2}} \frac{\partial}{\partial \theta}\left(h^{3} \frac{\partial p}{\partial \theta}\right)+h^{3} \frac{\partial^{2} p}{\partial z^{2}}=-12 \mu_{s} \Omega \frac{\partial h}{\partial \theta}+12 \mu \frac{\partial h}{\partial t} .
$$

where, $p$ is the pressure distribution of oil film, $R_{s}$ is the radius of the damper, $\mu_{s}$ is the oil viscosity, $h$ is the oil film thickness, $z$ is the axial displacement in the $z$ direction, $\Omega$ is precession angular velocity of the axis, and $\theta$ is the angular displacement from the maximum oil thickness position.

The oil film bearing forces can be obtained by using short bearing theory and the Sommerfeld transform, expressed as:

$$
\left\{\begin{array}{c}
F_{\mathrm{sx}}=-\frac{\mu_{s} R_{s} L^{3}}{c^{2} \sqrt{x^{2}+y^{2}}}\left[x\left(\dot{\varepsilon} I_{1}+\varepsilon \Omega I_{2}\right)-y\left(\dot{\varepsilon} I_{2}+\varepsilon \Omega I_{3}\right)\right] \\
F_{\mathrm{sy}}=-\frac{\mu_{s} R_{s} L^{3}}{c^{2} \sqrt{x^{2}+y^{2}}}\left[y\left(\dot{\varepsilon} I_{1}+\varepsilon \Omega I_{2}\right)+x\left(\dot{\varepsilon} I_{2}+\varepsilon \Omega I_{3}\right)\right]
\end{array} .\right.
$$

and:

$$
I_{1}=\int_{\theta_{1}}^{\theta_{1}+\pi} \frac{\cos ^{2} \theta}{(1+\varepsilon \cos \theta)^{3}} d \theta, I_{2}=\int_{\theta_{1}}^{\theta_{1}+\pi} \frac{\sin \theta \cos \theta}{(1+\varepsilon \cos \theta)^{3}} d \theta, I_{3}=\int_{\theta_{1}}^{\theta_{1}+\pi} \frac{\sin ^{2} \theta}{(1+\varepsilon \cos \theta)^{3}} d \theta
$$

where $\varepsilon=\frac{e_{B}}{c}$ is the journal eccentricity ratio, $e_{B}$ is the eccentricity of the journal and $\dot{\varepsilon}$ is the first derivative of journal eccentricity ratios with respect to time.

\subsection{Rolling Bearing Force Model}

In the process of rotation, the varying compliance vibration is caused by rolling bearing stiffness changing periodically. Based on the Hertzian contact theory [15], the supporting forces are given as:

$$
\left\{\begin{array}{c}
F_{b x}=\sum_{i=1}^{n} C_{b}\left[\left(x \cos \theta_{i}+y \sin \theta_{i}\right) \cos \alpha-\mu\right]^{3 / 2} \\
\cdot H\left[\left(x \cos \theta_{i}+y \sin \theta_{i}\right) \cos \alpha-\mu\right] \cos \theta_{i} \\
F_{b y}=\sum_{i=1}^{n} C_{b}\left[\left(x \cos \theta_{i}+y \sin \theta_{i}\right) \cos \alpha-\mu\right]^{3 / 2} \\
\cdot H\left[\left(x \cos \theta_{i}+y \sin \theta_{i}\right) \cos \alpha-\mu\right] \sin \theta_{i} \\
\theta_{i}=\frac{2 \pi}{n}(i-1)+\omega_{\text {cage }} t, \omega_{\text {cage }}=\left(\frac{R_{i}}{R_{i}+R_{o}}\right) \omega
\end{array}\right.
$$


In the formula, $F_{b x}, F_{b y}$ are supported forces of rolling bearing in the $X$ and $Y$ directions respectively, $C_{b}$ is the Hertz contact stiffness, $n$ is the number of rolling element, $\alpha$ is contact angle of rolling bearing, $\theta_{i}$ is the position angle of the $i$ th ball, $\omega_{\text {cage }}$ is the angular velocity of the cage, $R_{i}, R_{o}$ are the radius of the inner and outer ring raceway respectively, $\mu$ is the bearing clearance, $H$ is the heaviside function.

\subsection{The Optimal Latin Hyercube Design}

The Latin hyercube design is used to divide each dimension coordinate interval $\left[x_{k}^{\min }, x_{k}^{\max }\right]$, $k \in[1, n]$ in the $\mathrm{n}$-dimensional space into $\mathrm{m}$ intervals, each interval is $x_{k}^{i-1}, x_{k^{\prime}}^{i}, i \in[1, \mathrm{~m}]$. Randomly selected $\mathrm{m}$ points, and ensured that each level of a factor has been studied only once, leading to an $\mathrm{n}$-dimensional space, the sample number $\mathrm{m}$ of Latin hypercube design, marked as mxn LHD [16]. The optimal Latin hypercube design is improved at the foundation of random Latin hypercube design, so all the test points are distributed as evenly as possible in the design space, so as to further enhance its space filling and equalization. Figure 3 shows test points generated by random Latin hypercube design and test points generated by optimal Latin hypercube design, respectively.

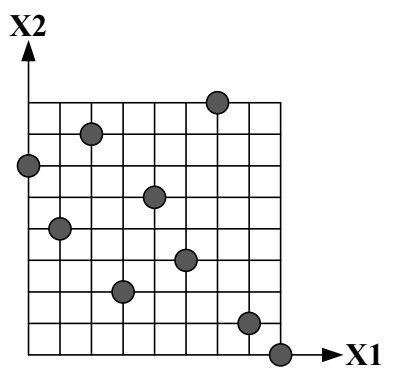

(a)

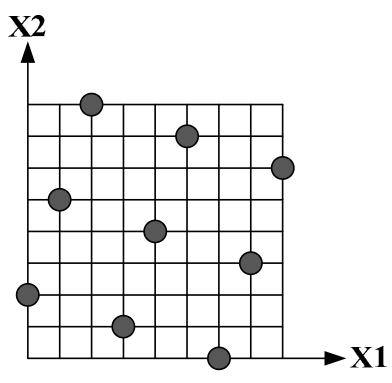

(b)

Figure 3. Latin Hypercube Design. (a) Random Latin hypercube and (b) optimal Latin hypercube.

\subsection{Non-Dominated Sorting Genetic Algorithm with Elite Strategy (NSGA-II)}

At present, the frequently used multi-objective algorithm is mainly divided into two kinds: one method is based on non-Pareto, the other is based on Pareto. For the method based on non-Pareto, such as a genetic algorithm VEGA based on vector evaluation, because of its high probability of local optimization, the performance still needs to be improved. While based on Pareto, NSGA-II is the most effective, it has improved at the foundation of the first-generation of NSGA [17]. The mian process is shown in Figure 4.

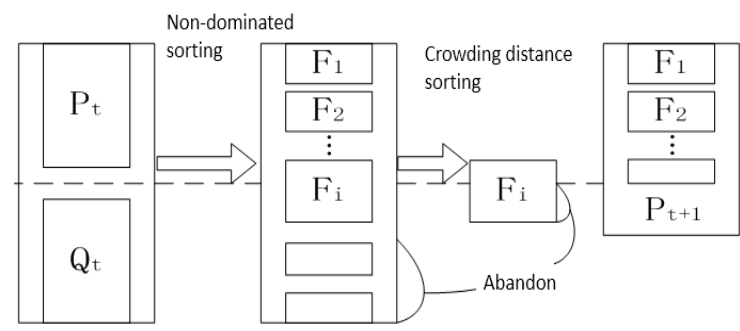

Figure 4. The main process of NSGA-II.

\section{Multi Objective Optimization of SFD Parameters}

\subsection{Initial Scheme and Experimental Design of SFD}

SFD can effectively reduces the vibration of the rotor system and restrain the nonlinearity caused by the misalignment coupling fault, in this paper, a variable-structured SFD is designed, which is shown in Figure 5, the SFD oil film gap $C_{S}$ and elastic support stiffness $K_{\mathrm{a}}$ are changed by adjusting the 
relative position of moving ring and length of elastic rod. The oil viscosity $\mu_{\mathrm{s}}$ also can be changed with the help of Magnetorheological Fluid. According to the structural characteristics of SFD, to realize the optimization of SFD structure parameters, and taking the SFD oil film gap $C_{S}$, elastic support stiffness $K_{\mathrm{a}}$ and oil viscosity $\mu_{\mathrm{s}}$ as variables, the changing range of various parameters are shown in Table 1.

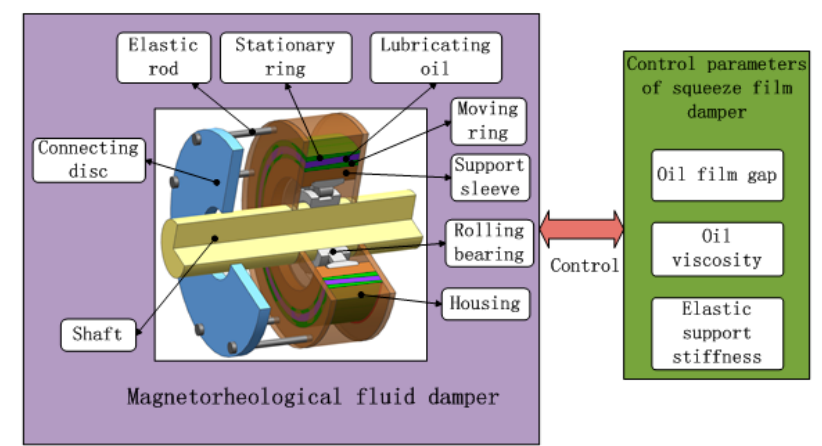

Figure 5. Schematic diagram of the variable-structured SFD.

Table 1. SFD Scheme structure variable.

\begin{tabular}{ccc}
\hline Design Parameters & Initial Value & The Changing Range/mm \\
\hline$C_{\mathrm{S}}(\mathrm{mm})$ & $8 \times 10^{-5}$ & $\left(4 \times 10^{-5}, 2 \times 10^{-4}\right)$ \\
$K_{\mathrm{a}}(\mathrm{N} \cdot \mathrm{m})$ & $3 \times 10^{6}$ & $\left(6 \times 10^{5}, 8 \times 10^{7}\right)$ \\
$\mu_{\mathrm{s}}(\mathrm{Pa} \cdot \mathrm{s})$ & 0.02 & $(0.005,0.01)$ \\
\hline
\end{tabular}

First, according to the changing range of the above mentioned variables and the optimal Latin hypercube design, the design matrix table of each parameter of SFD is obtained, and a total of 80 groups is obtained, while the design matrix of the SFD parameters is shown in Table 2.

Table 2. The design matrix of the SFD parameters.

\begin{tabular}{ccccc}
\hline Design Parameter & $\mathbf{1}$ & $\mathbf{2}$ & $\mathbf{3}$ & $\mathbf{8 0}$ \\
\hline$C_{\mathrm{S}}(\mathrm{mm})$ & $1.4 \times 10^{-4}$ & $1.1 \times 10^{-4}$ & $1.5 \times 10^{-4}$ & $1.8 \times 10^{-4}$ \\
$K_{\mathrm{a}}(\mathrm{N} \cdot \mathrm{m})$ & $6 \times 10^{6}$ & $8.7 \times 10^{6}$ & $3.9 \times 10^{6}$ & $4.8 \times 10^{6}$ \\
$\mu_{\mathrm{s}}(\mathrm{Pa} \cdot \mathrm{s})$ & $4.8 \times 10^{-2}$ & $2.3 \times 10^{-2}$ & $2.7 \times 10^{-2}$ & $4.6 \times 10^{-2}$ \\
\hline
\end{tabular}

Second, according to the optimized parameters of SFD in the design matrix of above experiment, a dynamic model of the SFD rotor rolling bearing system under three states is established: normal, misalignment fault, and the misalignment-rubbing coupling fault, while the model equations of motion are solved using the fourth-order Runge-Kutta method. The model parameters of the rotor shaft, SFD, ball bearing, rub-impact, and spline coupling are listed in Appendix A. Finally, the design parameters of SFD in Table 1 are used as inputs, while the calculated frequency amplitude is used as output, introducing into RBF neural network model, so as to establish an approximate model of SFD design parameters on the system frequency amplitude. Meanwhile, 10 sets of design parameters are selected and calculated by using the optimal Latin hypercube method, and the calculated results and prediction results of an approximate model are compared.

\subsection{Single Objective Parameter Optimization Analysis of the Normal Rotor System}

Figure 6 shows the system response characteristic of the system in the state of no fault, it can be found that spectrum diagram mainly manifested as the fundamental frequency vibration caused by the unbalance eccentricity. The motion of rotor is an ellipse, which means the system completes a single-period movement. 


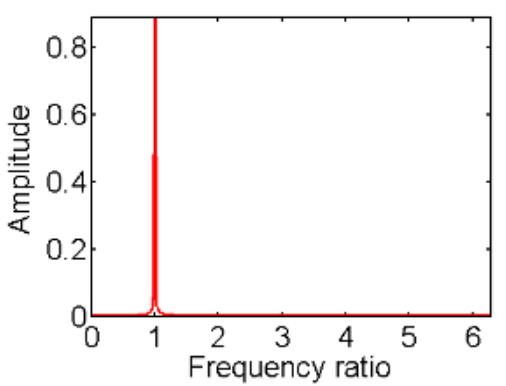

(a)

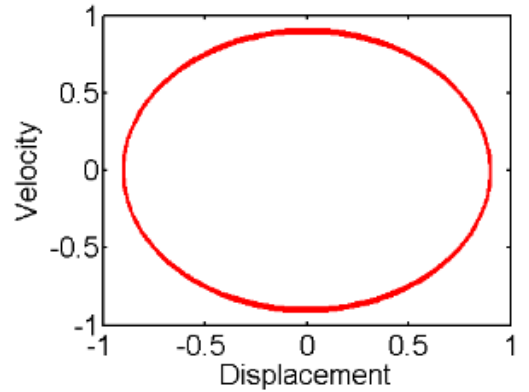

(b)

Figure 6. Dynamic behavior of the normal rotor system. (a) Spectrogram; (b) Phase Diagram.

Because of the vibration energy of normal system is mainly concentrated in the fundamental frequency, the optimization objective function is aiming at the minimum of its amplitude.

Figure 7 shows the calculation results of the 10 sets of normal system design parameters selected by the optimal Latin hypercube method and the comparison of the predicted results with the RBF approximation model. Because the fundamental frequency which caused by the rotating speed is the mainly response, which can get the average error value of the amplitude of one time frequency is $0.9 \%$, the maximum error value is $2.7 \%$. The error of the approximate model output variables can be controlled within $10 \%$, which shows that the approximate model has high precision, and can be used to optimize the SFD parameters of the normal rotor system.

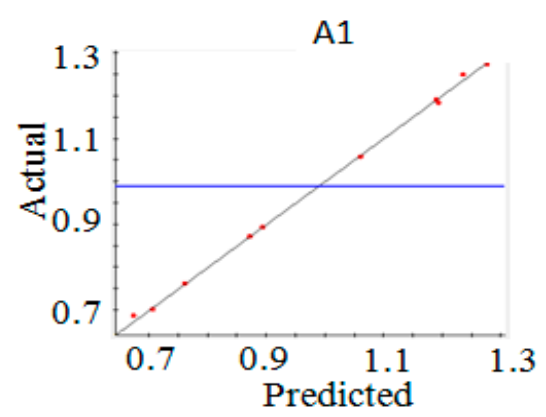

Figure 7. Comparison between calculated results and approximate model with no fault.

Figure 8 shows the optimization parameters of oil film gap $C_{\mathrm{S}}$, elastic support stiffness $K_{\mathrm{a}}$, and the influence of the oil viscosity of $\mu_{\mathrm{s}}$ on the amplitude of the fundamental frequency. The effect of three parameters to the system is strongly nonlinear, the $C_{S}$ has the most obvious effect.

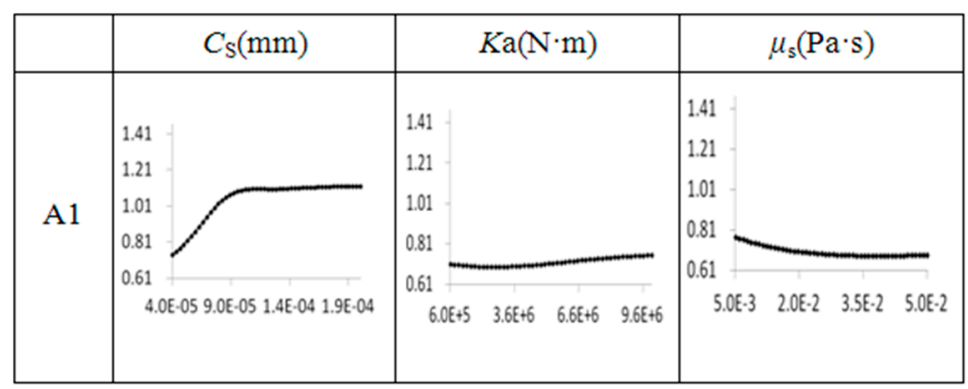

Figure 8. Effects of SFD design parameters on the amplitude of the fundamental frequency of the normal system. 
Sequential quadratic programming method (NLPQL) is introduced to optimize SFD parameters of normal system, the objective function is the amplitude of the fundamental frequency. The optimal solution for the design parameters of SFD can be solved as: $C_{\mathrm{S}}=4 \times 10^{-5} ; K_{\mathrm{a}}=8,008,000 ; \mu_{\mathrm{s}}=0.038$.

According to the design parameters, the numerical simulation of the normal SFD- rotor system is made, and the dynamic response spectrum of the system is obtained. After comparing Figures 6 and 9, it can be seen that, after optimization, the amplitude of the fundamental frequency of the system dynamics response decreases from 0.8913 to 0.6858 , while the amplitude is reduced by nearly $23 \%$.

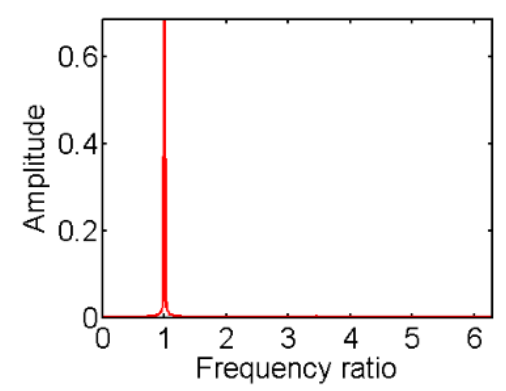

Figure 9. Spectrum diagram of optimized system with no fault.

\subsection{Analysis Results of Optimization Parameters of Rotor System with the Misalignment Fault}

In this section, the SFD optimization parameters of the rotor system with no fault are used as the initial calculated parameters of the SFD of rotor system under misalignment fault, to analyze the dynamic characteristics of the rotor system with misalignment, in which the misalignment value is $\mathrm{e}_{0}$ $=3 \times 10^{-4} \mathrm{~m}$.

Figure 10 shows the response characteristic of the system under the misalignment fault, from Figure 10a, it can be found that the fault makes the system produce $2 \times$ fundamental frequency, $3 \times$ fundamental frequency and other high frequency components, the VC frequency of rolling bearing has growth, while the frequency spectrum is mainly fundamental frequency, $2 \times$ fundamental frequency, and $3 \times$ fundamental frequency. The calculated results are in agreement with those in reference [12]. From Figure 10b, the phase diagram of the system is shown as a net 8-shaped figure, which means the system is in quasi periodic motion.

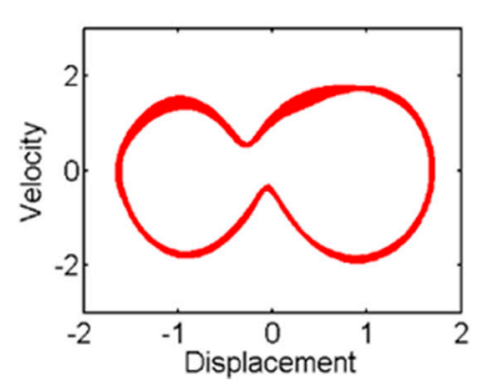

(a)

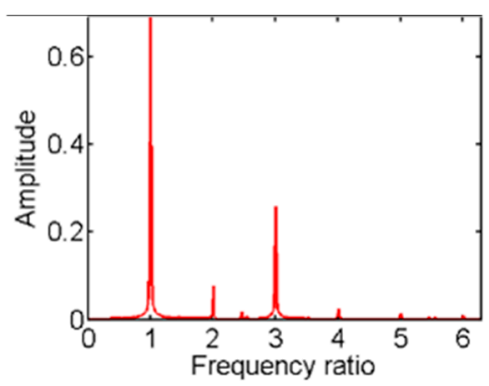

(b)

Figure 10. Dynamic behavior of system with misalignment fault. (a) Spectrum diagram; (b) Phase Diagram.

The energy of misalignment fault system is mainly concentrated in fundamental frequency, $2 \times$ fundamental frequency, $3 \times$ fundamental frequency components, the amplitude of fundamental frequency, $2 \times$ fundamental frequency, $3 \times$ fundamental frequency of the optimization objective function for the system response is minimal.

Figure 11 is a comparison of the calculated results of 10 sets of design parameters which were selected by using the optimal Latin hypercube method under misalignment fault and the predictive 
results of approximate model which is built by using the radial basis function (RBF). In this figure, the average error of fundamental frequency is $1.2 \%$, and the maximum error is $6.8 \%$; the average error of $2 \times$ fundamental frequency is $1.8 \%$, and the maximum error is $8.4 \%$; the average error of $3 \times$ fundamental frequency is $0.6 \%$, and the maximum error is $2.1 \%$. The error of the approximate model output variables can be controlled within $10 \%$, which shows that the approximate model has high accuracy and that it can be used to optimize the SFD parameters of the rotor system with misalignment-rubbing coupling faults.

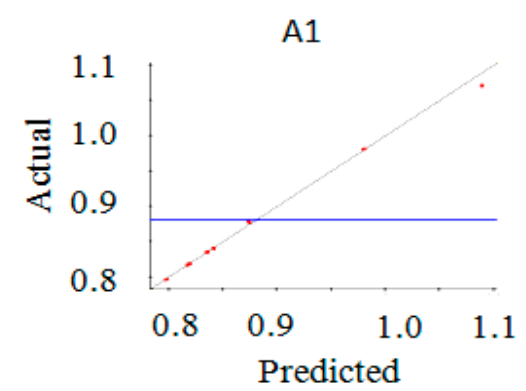

(a)

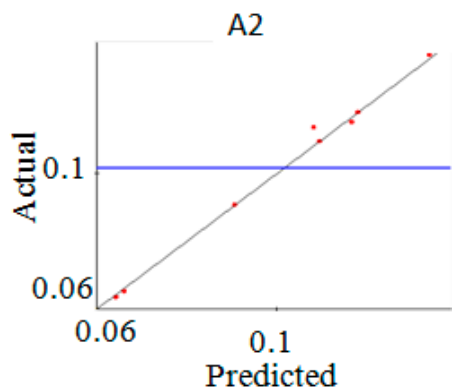

(b)

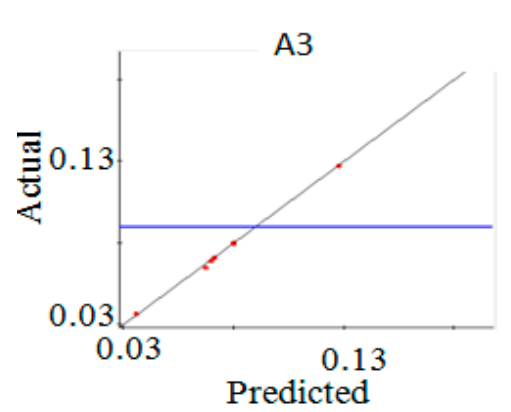

(c)

Figure 11. Comparison between calculated results and approximate model with misalignment fault.

(a) Fundamental frequency; (b) $2 \times$ fundamental frequency; (c) $3 \times$ fundamental frequency.

Figure 12 shows the optimization parameters of the oil film gap $C_{S}$, elastic support stiffness $K_{a}$ and the impact of the viscosity of the lubricating oil $\mu_{\mathrm{s}}$ on the amplitude of fundamental frequency, $2 \times$ fundamental frequency and $3 \times$ fundamental frequency. From Figure 12, it can be seen that the effect of the three parameters on the system is nonlinear. As a result, fundamental frequency, $3 \times$ fundamental frequency mainly influences the oil film gap $C_{S}$; and the $2 \times$ fundamental frequency is mainly influenced by the support stiffness $K_{\mathrm{a}}$.

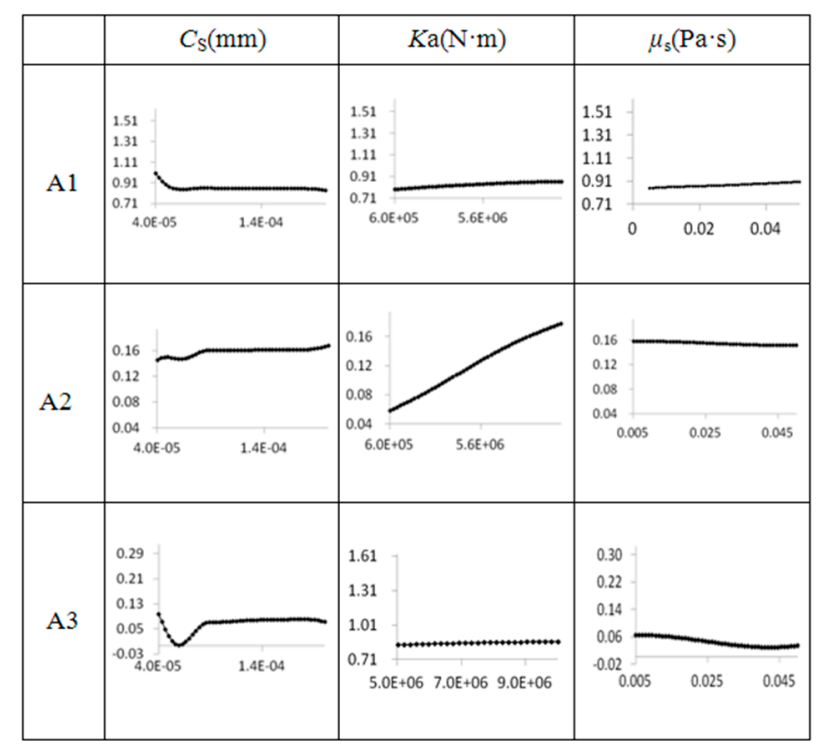

Figure 12. Effect of SFD design parameters on the amplitude of the fundamental, $2 \times$ fundamental and $3 \times$ fundamental frequency of the system with misalignment fault.

The SFD parameters of rotor system under the misalignment fault is optimized by using NSGA-II, the parameter configuration of the algorithm is as follows: the population size is 40, algebra is 200, the crossover rate is 0.9 , cross distribution index is 10 , variation distribution index is 20 . On these grounds, the optimal solution of SFD is $C_{S}=2 \times 10^{-4} ; K_{\mathrm{a}}=6 \times 10^{5} ; \mu_{\mathrm{s}}=5 \times 10^{-3}$. 
According to the design parameters, the numerical simulation of the SFD- rotor system is carried out again, and the dynamic response spectrum of the system is obtained, as shown in Figure 13, and compared with the spectrum diagram in Figure 10a before optimization. It can be concluded that after optimization, the amplitude of fundamental frequency of system dynamics response decreases from 0.6916 to 0.3913 , the amplitude decreases by about $43.4 \%$; the amplitude of $2 \times$ fundamental frequency decreases from 0.0766 to 0.0555 , the amplitude decreases by about $27.5 \%$; the amplitude of $3 \times$ fundamental frequency decreases from 0.2591 to 0.0863 , and the amplitude decreases by about $66.7 \%$.

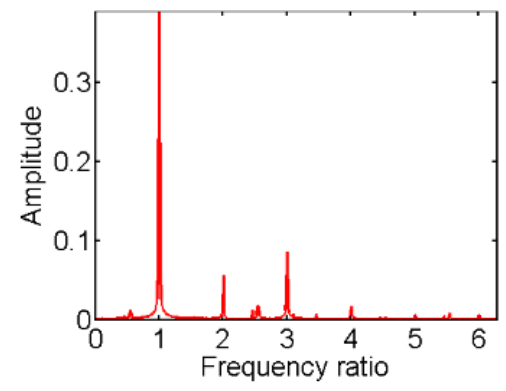

Figure 13. Spectrum diagram of SFD optimized system with misalignment fault.

\subsection{Analysis Results of Optimization Parameters of Rotor System with the Misalignment-Rubbing Coupling Fault}

In this section, the SFD optimization parameters of the rotor system with the fault of misalignment referred in front section are used as the initial calculated parameters of the rotor system SFD, and the dynamic characteristics of the rotor system with misalignment-rubbing coupling faults are analyzed.

Figure 14 shows the system response characteristic of the system with misalignment-rubbing coupling faults. The fundamental frequency, $2 \times$ fundamental frequency, and $3 \times$ fundamental frequency are mainly shown in spectrum. Compared with a spectrum diagram with a misalignment fault, the rubbing coupling faults made the amplitude of fundamental frequency of the system increase a lot, and made the amplitude of the $2 \times$ fundamental frequency, $3 \times$ fundamental frequency change a small amount, and brought about an emergency of the continuous spectrum. However, the impact is modest, and the energy is low, meaning the calculated result obtained in this paper is in agreement with the experimental result of reference [18]. As seen in Figure 14a,b the phase diagram is a net 8-shaped figure, which means the system is in quasi periodic motion.

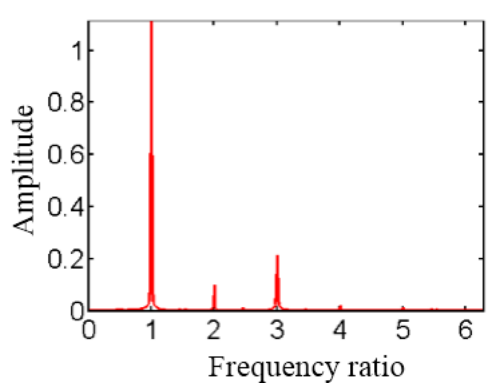

(a)

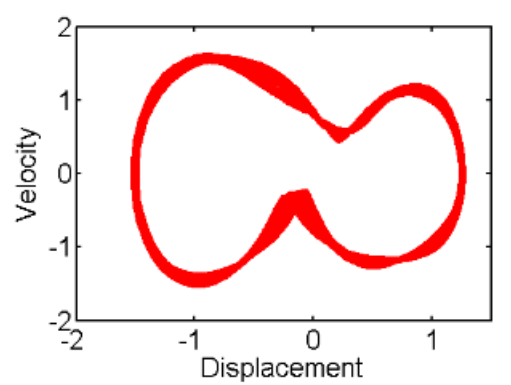

(b)

Figure 14. Dynamic behavior of system with the misalignment-rubbing coupling fault. (a) Spectrum diagram; (b) Phase diagram.

The energy of the misalignment-rubbing coupling faults is mainly concentrated in fundamental frequency, $2 \times$ fundamental frequency, and $3 \times$ fundamental frequency components, thus the amplitude of the fundamental frequency, $2 \times$ fundamental frequency, and $3 \times$ fundamental frequency of optimization objective function of the system responses is minimal. 
Figure 15 is a comparison of the calculated results of 10 sets of design parameters which selected by using optimal Latin hypercube method under misalignment fault and the predictive results of approximate model which is built by using the radial basis function (RBF). Here, the average error of fundamental frequency is $0.56 \%$, and the maximum error is $1.44 \%$; the average error of $2 \times$ fundamental frequency is $1.47 \%$, and the maximum error is $4.10 \%$, the average error of $3 \times$ fundamental frequency is $0.47 \%$, and the maximum error is $1.2 \%$. The error of the approximate model output variables can be controlled within $10 \%$, which shows that the approximate model has high accuracy and can be used to optimize the SFD parameters of the rotor system with misalignment-rubbing coupling faults.

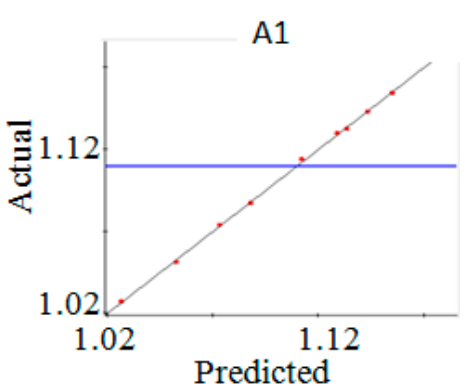

(a)

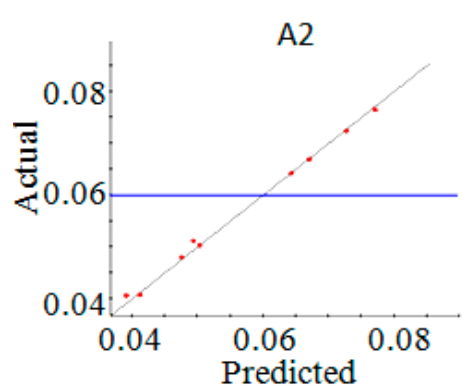

(b)

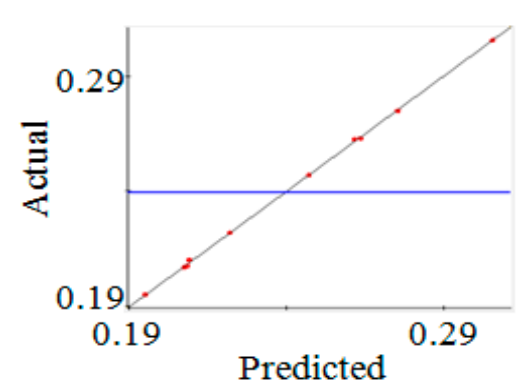

(c)

Figure 15. Comparison between calculated results and approximate model with the misalignment-rubbing coupling fault. (a) fundamental frequency; (b) $2 \times$ fundamental frequency; (c) $3 \times$ fundamental frequency.

Figure 16 shows the optimization parameters of the oil film gap $C_{S}$, elastic support stiffness $K_{\mathrm{a}}$ and the impact of the viscosity of the lubricating oil $\mu_{\mathrm{s}}$ on the amplitude of the fundamental, $2 \times$ fundamental and $3 \times$ fundamental frequency. From Figure 16, it can be seen that the impact of the three parameters on the system is nonlinear. Here, $C_{S}$ has a great influence on $2 \times$ fundamental frequency, and basically it increases linearly in the early stage. For elastic support stiffness $K_{\mathrm{a}}$, when its value increases, the amplitude of fundamental and $3 \times$ fundamental frequency increases gradually, while the amplitude of $2 \times$ fundamental frequency is shown as the opposite trend. For the viscosity of the lubricating oil $\mu_{\mathrm{s}}$, when its value increases, the amplitude of fundamental frequency and $3 \times$ fundamental frequency decrease gradually, while the trend of the amplitude of $2 \times$ fundamental frequency showed decreases at first, and increases at the end.

The SFD optimization parameter of rotor system is carried out by using NSGA-II, and the parameter configuration of NSGA-II algorithm is same as the parameter configuration with misalignment faults. The optimal solution of SFD is obtained as $C_{S}=8 \times 10^{-5} ; K_{\mathrm{a}}=6 \times 10^{5} ; \mu_{\mathrm{s}}=5 \times 10^{-2}$.

According to the design parameters, the numerical simulation of the SFD-rotor system is carried out under the state of the misalignment-rubbing coupling fault, and the dynamic response spectrum of the system is obtained, compared with spectrum in Figures 14a and 17, it can be seen that after being optimized, the amplitude of fundamental frequency of system dynamics response decreases from 1.116 to 1.033 , the amplitude decreases by about $7.4 \%$; the amplitude of $2 \times$ fundamental frequency decreases from 0.099 to 0.048 , the amplitude reduces by about $51.5 \%$, the amplitude of $3 \times$ fundamental frequency decreases from 0.214 to 0.178 , and the amplitude reduces by about $16.8 \%$. 


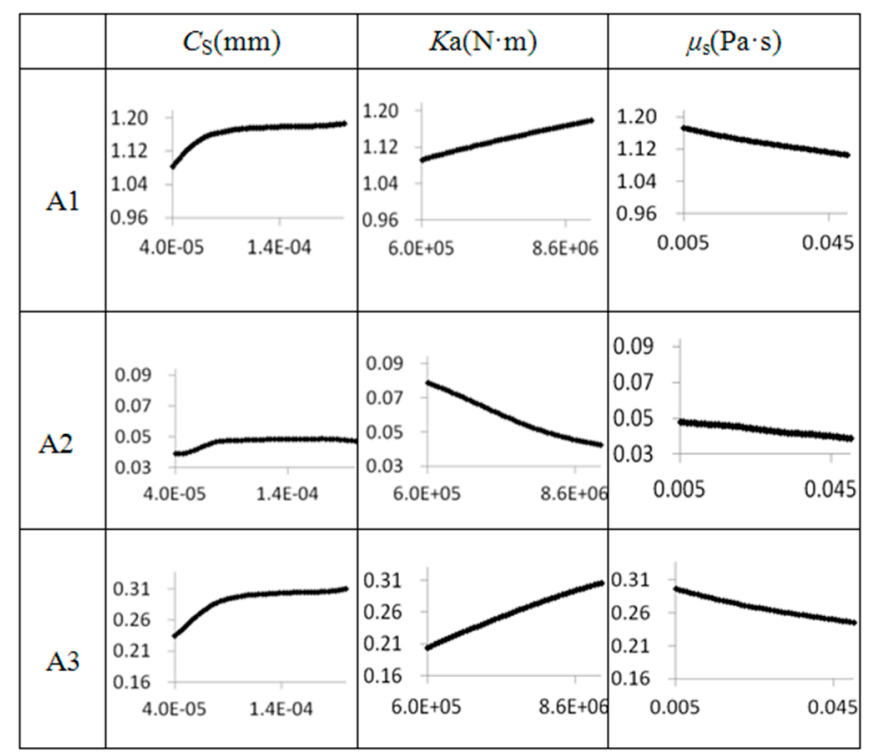

Figure 16. Effect of SFD design parameters on the amplitude of the fundamental, $2 \times$ fundamental and $3 \times$ fundamental frequency of the system with the misalignment-rubbing coupling fault.

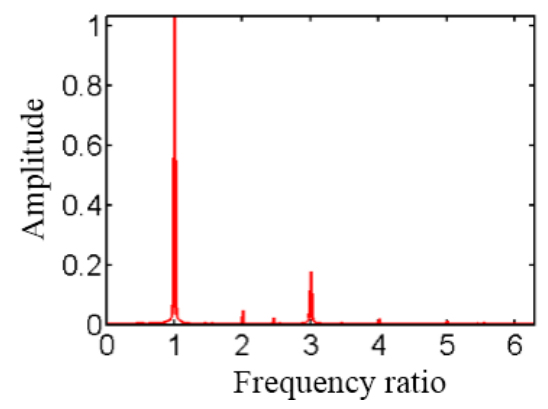

Figure 17. Spectrum diagram of SFD optimized system with the misalignment-rubbing coupling fault.

\subsection{Comparison Results of SFD Parameters of the Rotor System under Three Conditions}

Table 3 is SFD optimization parameters with normal, misalignment faults, and misalignment-rubbing coupling faults. It can be found that SFD optimization parameters are different under various conditions. The optimal value of the oil film gap is at the minimum level under the normal condition, and the optimal value is increased when the misalignment fault is considered. While the optimal value is relatively reduced, the misalignment-rubbing coupling faults are considered. For the effect of elastic support stiffness, the optimal value is the largest under the normal state, and the optimal value is the same under the state of the misalignment fault and the misalignment-rubbing coupling fault, while the optimal value reduces under the normal state. For the viscosity of the oil lubrication, the optimal value is the largest under the state of misalignment-rubbing coupling fault, while the optimal value reduces under the state of misalignment fault, and the optimal value relatively increases under the normal state. The reason that SFD optimization parameters are different under various conditions may be that various conditions have different influences on the rotor system, resulting in the change of dynamic characteristics of the rotor system. 
Table 3. SFD parameters of the rotor system under three conditions.

\begin{tabular}{cccc}
\hline System Status & $C_{\mathbf{S}}(\mathbf{m m})$ & $K_{\mathbf{a}}(\mathbf{N} \cdot \mathbf{m})$ & $\mu_{\mathbf{s}}(\mathbf{P a} \cdot \mathbf{s})$ \\
\hline Before optimization & $8 \times 10^{-5}$ & $3 \times 10^{6}$ & 0.02 \\
\hline $\begin{array}{c}\text { No fault } \\
\text { (after optimization) }\end{array}$ & $4 \times 10^{-5}$ & $8 \times 10^{6}$ & 0.038 \\
\hline $\begin{array}{c}\text { Misalignment faults } \\
\text { (after optimization) }\end{array}$ & $2 \times 10^{-4}$ & $6 \times 10^{5}$ & $5 \times 10^{-3}$ \\
\hline $\begin{array}{c}\text { Misalignment-rubbing coupling faults } \\
\text { (after optimization) }\end{array}$ & $8 \times 10^{-5}$ & $6 \times 10^{5}$ & $5 \times 10^{-2}$ \\
\hline
\end{tabular}

\section{Conclusions}

In this paper, the vibration attenuation effects of the oil film gap $\left(C_{S}\right)$, elastic support stiffness $\left(K_{\mathrm{a}}\right)$ and the lubricating oil viscosity $\left(\mu_{\mathrm{s}}\right)$ of SFD selected by using the multi-objective optimization method on the dynamic responses of the faulted system were analyzed. Some conclusions drawn from the study can be summarized as follows:

(1) For the vibration responses caused by the system with misalignment fault, three variable parameters are strongly nonlinear, in which $K_{\mathrm{a}}$ has obvious impact on fundamental, $2 \times$ fundamental and $3 \times$ fundamental frequency, $C_{S}$ and $\mu_{\mathrm{s}}$ are weak., For the vibration responses caused by a misalignment-rubbing coupling faults system, in which $C_{\mathrm{S}}$ has a remarkable effect on fundamental frequency, $\mu_{\mathrm{s}}$ and $K_{\mathrm{a}}$ are sensitive to $2 \times$ fundamental and $3 \times$ fundamental frequency, respectively.

(2) Optimizing the parameters of SFD have an effect on vibration control and stability of the rotor system with varied faults by using NLPQL and NSGA-II algorithm. The vibration responses of the system with the optimization parameters are shown as: With no fault, the amplitude of fundamental frequency decreased by $23 \%$. With the misalignment fault, the amplitude of the fundamental frequency decreased by $43.4 \%$, the amplitude of $2 \times$ fundamental frequency decreased by $27.5 \%$, while the amplitude of $3 \times$ fundamental frequency decreased by $66.7 \%$. With the misalignment-rubbing coupling fault, the amplitude of fundamental frequency decreased by $7.4 \%$, the amplitude of $2 \times$ fundamental frequency decreased by $51.5 \%$, and the amplitude of $3 \times$ fundamental frequency decreased by $16.8 \%$. Overall, the feasibility of the optimization method of the variable-structured SFD operational parameters for the faulted rotor system was verified.

(3) The research results presented in the paper can provide helpful guidance for the design of the SFD and will be validated by experiments in the next planned step.

Author Contributions: L.M. developed the model and conducted the analyses; J.W. wrote and revised the paper; G.Z. contributed analysis tools.

Funding: This work is supported by the National Natural Science Foundation of China (No. 51705355).

Conflicts of Interest: The authors declare no conflict of interest.

\section{Appendix A}

$$
\begin{gathered}
\mathrm{M}=\operatorname{diag}\left(M_{\mathrm{s} 1}, M_{\mathrm{s} 1}, M_{\mathrm{d} 1}, M_{\mathrm{d} 1}, M_{\mathrm{s} 2}, M_{\mathrm{s} 2}, M_{\mathrm{s} 3}, M_{\mathrm{s} 3}, M_{\mathrm{s} 4}, M_{\mathrm{s} 4}, M_{\mathrm{s} 5}, M_{\mathrm{s} 5}, M_{\mathrm{d} 2}, M_{\mathrm{d} 2}, M_{\mathrm{s} 6}, M_{\mathrm{s} 6}, M_{\mathrm{o} 1}, M_{\mathrm{o} 1}, M_{\mathrm{o} 2}, M_{\mathrm{o} 2}\right) \\
\mathrm{C}=\operatorname{diag}\left(C_{\mathrm{z}}, C_{\mathrm{z}}, C_{\mathrm{p}}, C_{\mathrm{p}}, C_{\mathrm{z}}, C_{\mathrm{z}}, 0,0,0,0, C_{\mathrm{z}}, C_{\mathrm{z}}, C_{\mathrm{p}}, C_{\mathrm{p}}, C_{\mathrm{z}}, C_{\mathrm{z}}, 0,0,0,0\right)
\end{gathered}
$$




$$
\mathrm{K}=\left[\begin{array}{ccccccccccc}
k & 0 & -k & 0 & 0 & 0 & 0 & 0 & 0 & 0 & 0 \\
& k & 0 & -k & 0 & 0 & 0 & 0 & 0 & 0 & 0 \\
& & 2 k & 0 & -k & 0 & 0 & 0 & 0 & 0 & 0 \\
& & & \cdots & & & & & & & \\
& & & & 2 k & 0 & -k & 0 & 0 & 0 & 0 \\
& & & & & k & 0 & -k & 0 & 0 & 0 \\
& & & & & & k & 0 & -k & 0 & 0 \\
& & & & & & k_{a} & 0 & 0 & 0 \\
& & & & & & & & k_{a} & 0 & 0 \\
\text { sym } & & & & & & & & k_{a} & 0 \\
& & & & & & & & & k_{a}
\end{array}\right]
$$

Table A1. The model parameters of rotor shaft.

\begin{tabular}{cc}
\hline Parameters & Values \\
\hline$M_{\mathrm{s} 1}=M_{\mathrm{s} 2}=M_{\mathrm{s} 3}=M_{\mathrm{s} 4}=M_{\mathrm{s} 5}=M_{\mathrm{s} 6}(\mathrm{~kg})$ & 1 \\
$M_{\mathrm{d} 1}, M_{\mathrm{d} 2}(\mathrm{~kg})$ & 15,12 \\
$K(\mathrm{~N} / \mathrm{m})$ & $2.5 \times 10^{7}$ \\
$e_{1}, e_{2}(\mathrm{~m})$ & $1 \times 10^{-5}, 5 \times 10^{-6}$ \\
$C_{\mathrm{z}}, C_{\mathrm{p}}(\mathrm{N} \cdot \mathrm{s} / \mathrm{m})$ & 2100,1500 \\
\hline
\end{tabular}

Table A2. The model parameters of SFD.

\begin{tabular}{cc}
\hline Parameters & Values \\
\hline$R_{\mathrm{s}}, L(\mathrm{~m})$ & $0.03,0.011$ \\
\hline
\end{tabular}

Table A3. The model parameters of ball bearing.

\begin{tabular}{cc}
\hline Parameters & Values \\
\hline$n$ & 7 \\
$C_{\mathrm{b}}\left(\mathrm{N} / \mathrm{m}^{1.5}\right)$ & $5 \times 10^{9}$ \\
$\mu(\mathrm{m})$ & $3 \times 10^{-6}$ \\
\hline
\end{tabular}

Table A4. The model parameters of rubbing.

\begin{tabular}{cc}
\hline Parameters & Values \\
\hline$k_{s}(\mathrm{~N} / \mathrm{m})$ & $5 \times 10^{7}$ \\
$\delta(\mathrm{m})$ & $1 \times 10^{-5}$ \\
$f$ & 0.1 \\
\hline
\end{tabular}

Table A5. The model parameters of spline coupling.

\begin{tabular}{cc}
\hline Parameters & Values \\
\hline$z$ & 14 \\
$L_{i}, R(\mathrm{~m})$ & $3.07 \times 10^{-3}, 6.95 \times 10^{-2}$ \\
$T(\mathrm{~N} \mathrm{~m})$ & 1000 \\
\hline
\end{tabular}

\section{References}

1. Olove, B. Non-linear behavior of a flexible shaft. SFD 1997, 206, 255-260.

2. Humes, B. The Non-Linear Performance of Squeeze Film Damper; University of Sussex: Brighton, UK, 1977.

3. Copper, S. Preliminary investigation of oil film for the control of vibration. Mech. Eng. 1963, 5, 305-315.

4. Ruggiero, A.; D'Amato, R.; Magliano, E.; Kozak, D. Dynamical simulations of a flexible rotor in cylindrical uncavitated and cavitated lubricated journal bearings. Lubricants 2018, 6, 40. [CrossRef] 
5. Wang, J.; Zhang, J.; Ma, L.; Yu, Y. Study on the Effect Mechanism of MRD to Rotor System. Appl. Sci. 2019, 9, 2247. [CrossRef]

6. Chang-Jian, C.W. Non-linear dynamic analysis of a HSFD mounted gear-bearing system. Nonlinear Dyn. 2010, 62, 333-347. [CrossRef]

7. Chang-Jian, C.W.; Kuo, J.K. Bifurcation and chaos for porous squeeze film damper mounted rotor-bearing system lubricated with micropolar fluid. Nonlinear Dyn. 2009, 58, 697-714. [CrossRef]

8. Chang-Jian, C.W.; Yau, H.T.; Chen, J.L. Nonlinear dynamic analysis of a hybrid squeeze-film damper-mounted rigid rotor lubricated with couple stress fluid and active control. Appl. Math. Model. 2010, 34, 2493-2507. [CrossRef]

9. Inayat-Hussain, J.I. Bifurcations of a flexible rotor response in squeeze-film dampers without centering springs. Solitons Fractals 2005, 24, 583-596. [CrossRef]

10. Inayat-Hussain, J.I. Bifurcations in the response of a flexible rotor in squeeze-film dampers with retainer springs. Solitons Fractals 2009, 39, 519-532. [CrossRef]

11. Qin, W.; Zhang, J.; Wang, H. Response and bifurcation of rotor with squeeze film damper supported on elastic foundation. J. Northwestern Polytech. Univ. 2006, 24, 245-248.

12. Yuan, H.; Wang, Z.; Wen, B. Analysis on stability of dual-discs rub-impact rotor with consideration of casing elasticity. J. Vib. Shock. 2010, 29, 52-54.

13. Zhao, G.; Liu, Z.; Ye, J. Dynamic behavior of a rotor-misaligned spline coupling system. J. Vib. Shock 2009, 28, $78-82$.

14. Wen, B.; Gu, J.; Xia, S. The Advanced Rotor Dynamics; China Machine Press: Beijing, China, 2000.

15. Cheng, M.; Meng, G. Dynamic analysis of a rotor-ball bearing nonlinear system with Alford force. China Mech. Eng. 2011, 22, 2806-2812.

16. Park, J.S. Optimal latin-hypercube designs for computer experiments. J. Stat. Plan. Inference 1994, 39, 95-111. [CrossRef]

17. Lei, D.; Yan, X. Multi-Objective Intelligent Optimization Algorithm and Its Application; Science Press: Beijing, China, 2009.

18. Li, X. Dynamic Analysis and Identification for Rotor Misalignment-Rubbing Coupling Faults; Nanjing University of Aeronautics and Astronautics: Nanjing, China, 2009. 